Epidemiology and Infection

cambridge.org/hyg

\section{From the Field}

Cite this article: Turner S (2020). Numerous outbreaks amongst homeless and injection drug-using populations raise concerns of an evolving syndemic in London, Canada. Epidemiology and Infection 148, e160, 1-7. https://doi.org/10.1017/S0950268820001260

Received: 22 January 2020

Revised: 18 April 2020

Accepted: 5 June 2020

\section{Key words:}

Community outbreaks; hepatitis A; hepatitis C; HIV/AIDS; necrotising fasciitis (group A streptococcus)

Author for correspondence:

S. Turner, E-mail: stephen.turner@mlhu.on.ca

\section{Numerous outbreaks amongst homeless and injection drug-using populations raise concerns of an evolving syndemic in London, Canada}

\section{S. Turner (10)}

Environmental Health and Infectious Diseases Division, Middlesex-London Health Unit, London, Ontario, Canada

\begin{abstract}
London, Ontario is a mid-sized Canadian city which appears to be experiencing a syndemic predominately amongst its marginalized populations. Since 2014, rates of HIV, hepatitis A (HAV), hepatitis C (HCV), and invasive group A streptococcal disease have climbed well above provincial rates amid increasing use of injection drugs. Rates of infective endocarditis have also been on the rise. Extensive public health and community-based efforts were taken in response to these concurrent outbreaks. These efforts included establishing improved client care pathways, creating specialized teams to engage underhoused clients, providing mass immunization, and developing new health promotion campaigns. Rates of HIV and HAV were subsequently controlled locally while rates of HCV, iGAS and infective endocarditis remain high within the community and throughout the province.
\end{abstract}

London, Ontario, Canada is a city with a population of approximately 400000 experiencing multiple concurrent outbreaks disproportionately affecting marginalized residents. Rates of hepatitis $\mathrm{C}(\mathrm{HCV})$, invasive group A streptococcal disease (iGAS), HIV and hepatitis A (HAV) have significantly exceeded the provincial rates of these diseases in Ontario, most notably over the past 5 years [Fig. 1]. Rates of infective endocarditis have also been increasing. Those who are homeless, underhoused or who use injection drugs have shown to be at the highest risk for these infections.

Despite the region's relative affluence, $11.6 \%$ of the population lives below the low-income cut-off [1], and the 2018 point-in-time homelessness enumeration identified 406 individuals and families living without shelter $(\sim 0.1 \%$ pop. $)$ [2]. Further, harm reduction agencies have estimated that in London, there are approximately 6000 people who inject drugs (PWID) ( $\sim 1.5 \%$ pop.) [3]. It was striking to note that well over half of the cases identified in these outbreaks were representatives of these groups raising concerns that this might be evidence of the development of a local syndemic.

A syndemic is characterized by 'the synergistic interaction of two or more coexistent diseases and resultant excess burden of disease' and recognizes the role biological or social factors play in exacerbating such outbreaks [4]. An example of a biological synergism is seen with the interaction between HIV and tuberculosis (TB) where one of the highest risk factors for conversion from latent TB to active disease is seen when the patient is also infected with HIV [5]. Social factors contributing to poorer health outcomes associated with chronic diseases such as malnutrition and diabetes may also exhibit synergistic effects.

The HCV and HIV outbreaks in London described herein appear to take on the behaviour of a syndemic. As the number of emergency department (ED) visits for opioid overdoses increased between 2003 and 2006, an increase in the rate of HCV closely followed. When a rise in ED visits was later noted between 2015 and 2018, there was a concurrent increase in the number of new HIV cases reported. It could be argued that the relationship between the increase in the rate of injection drug use and these blood-borne infections was intertwined. The HCV and HIV infection rates would not likely be nearly as high if not for the presence of substance addictions. In studying reported risk factors, the question arose, could the HAV and iGAS outbreaks also be related to injection drug use or homelessness?

Between 2005 and 2009, HCV rates in London began to increase from 32 cases per 100000 to 55 cases per 100000 population. Since 2006, the rate has consistently been significantly higher than the average provincial rate of 32 cases per 100000 [Fig. 2]. As HCV infections have strong associations with the sharing of injection drug equipment [6], this increase may have been an early indicator of an increase in injection drug use in the region. The increase in the number of ED admissions for opioid toxicity between 2003 and 2009 appears to further support this conclusion [Fig. 3].

In 2014, a sharp increase occurred in the number of new cases of HIV within the Middlesex-London region [Fig. 4]. Prior to that year, the rate of HIV in the region had consistently been below that of the province, but by 2016, both the local rate and number of new 


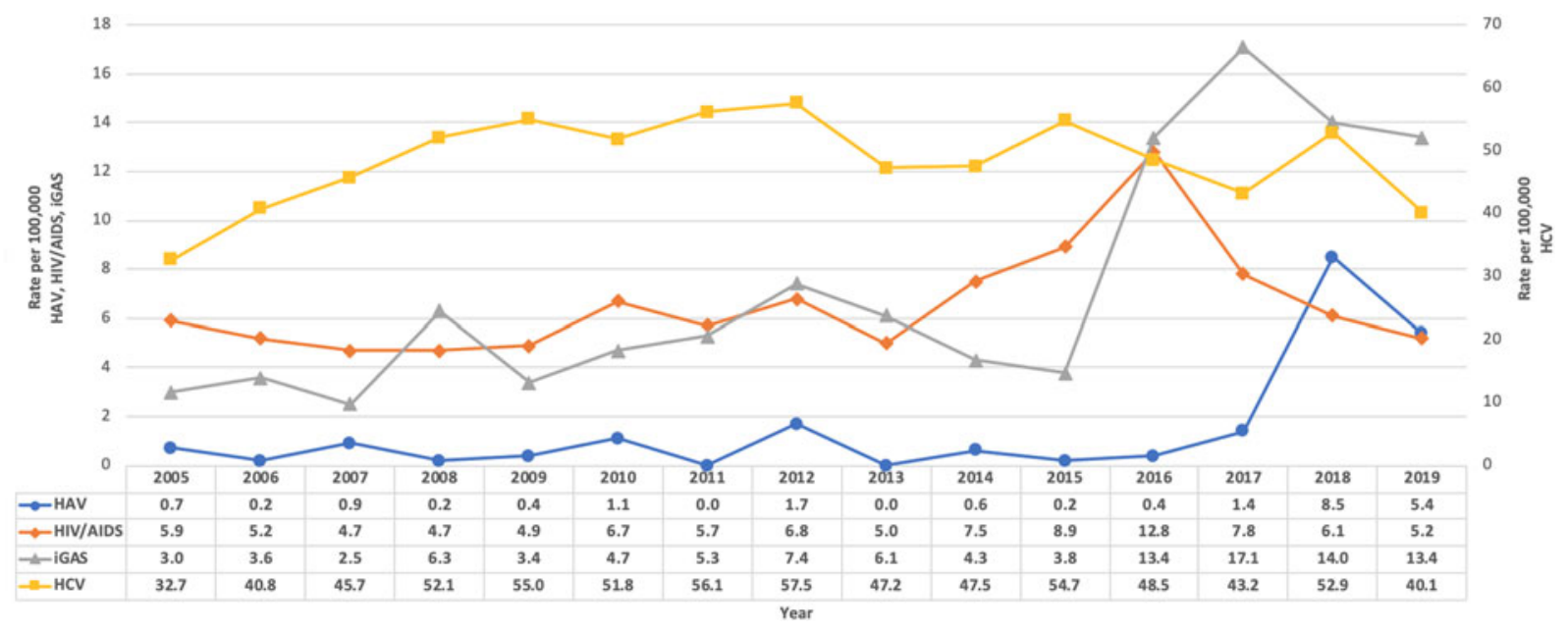

Fig. 1. Rate of hepatitis A (HAV), human immunodeficiency virus/acquired immune deficiency syndrome (HIV/AIDS), invasive group A streptococcus (iGAS) and hepatitis C (HCV) infections reported per 100000 population, Middlesex-London, 2005-2019. Data source: Public Health Ontario Infectious Disease Query: MLHU: Case counts and crude rates by public health unit and year. Toronto ON: Ontario Agency for Health Protection and Promotion; 2020 Apr 10 [cited 2020 Apr 12]. Available from https://www.publichealthontario.ca/data-and-analysis/infectious-disease/id-query.

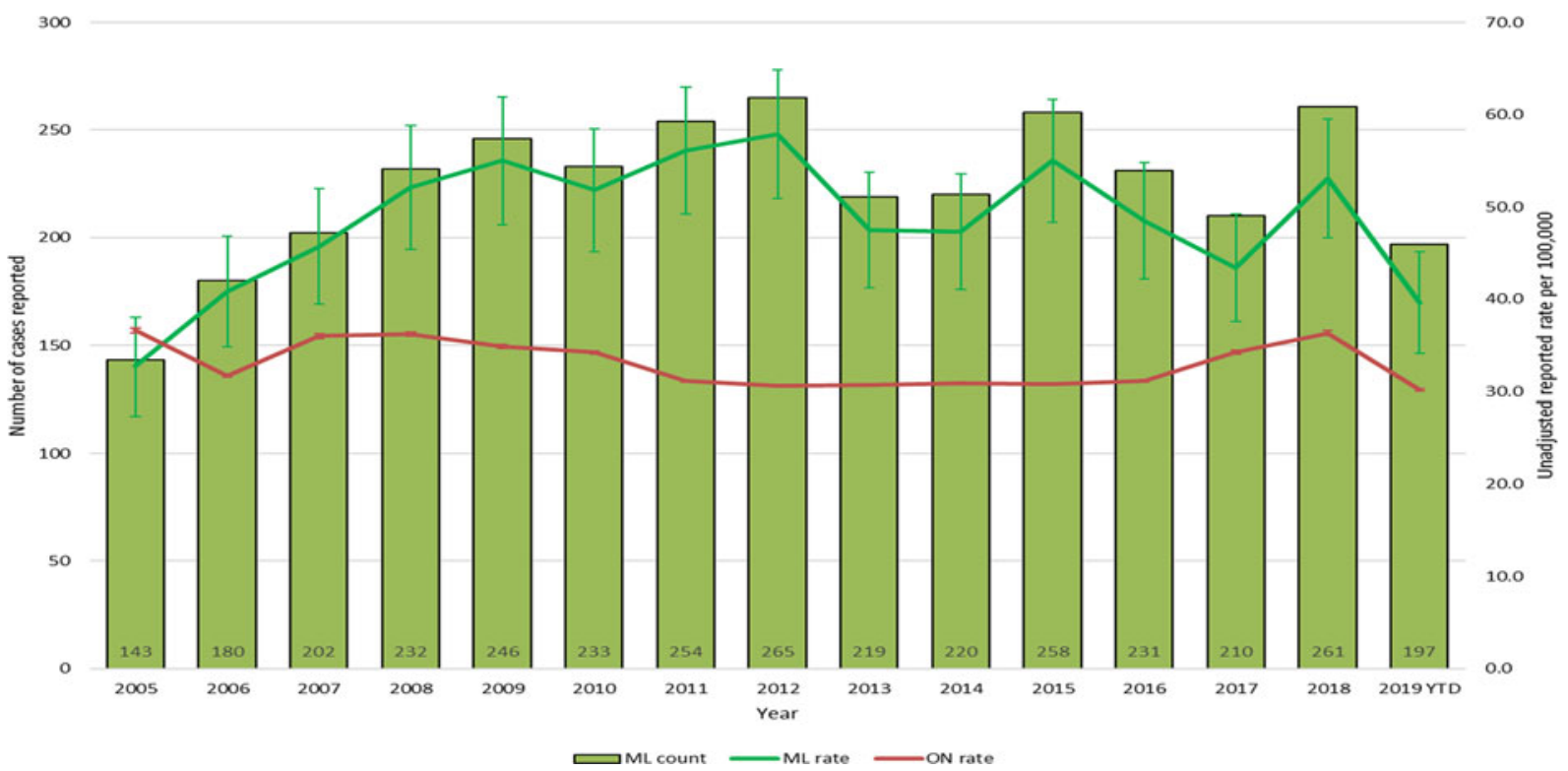

Fig. 2. Count and rate of hepatitis C cases, Middlesex-London and Ontario, 2005-2019 (Note: Ontario rate excludes Middlesex-London cases). Data source: Public Health Ontario Infectious Disease Query: MLHU: Case counts and crude rates by public health unit and year. Toronto ON: Ontario Agency for Health Protection and Promotion; 2019 Dec 18 [cited 2019 Dec 20]. Available from https://www.publichealthontario.ca/data-and-analysis/infectious-disease/id-query.

infections had more than doubled, prompting the region's Medical Officer of Health to identify the situation as a 'local public health emergency' [7]. At that time, $74 \%$ of all new cases of HIV reported having used injection drugs [3]. In studying the HIV outbreak, it was also noted that cases of infective endocarditis associated with injection drug use had been steadily increasing over the past decade, as well [Fig. 5].

That same year, a marked increase was also observed in the rate of iGAS infections. From 2015 to 2016, the local rate of iGAS more than tripled from four cases per 100000 to 12.5 cases per 100000 . By 2017, it had increased further to 17 cases per 100000 [Fig. 6]. Amongst cases reported between 2017 and 2019, 52\% were identified as being underhoused and/or using injection drugs. Emm type analysis also initially appeared to show some evidence of linkage between underhoused cases; however, that linkage became weaker over time [8].

In 2018, the region experienced an outbreak of HAV (Fig. 7). On average, the Middlesex-London region reports two cases of travel-related HAV each year and community transmission is very rare. Between July 2018 and July 2019, 68 HAV cases were reported of which only two were travel-related. Of the non-travel-related cases, two-thirds reported drug use, 39\% reported using injection drugs and $36 \%$ reported being underhoused (cases were able to report multiple risk factors). All cases involved in the outbreak were identified to have contracted the same genotype of HAV that had been identified in other outbreaks within Ontario indicating evidence of some level of intraprovincial migration within this community [9]. 


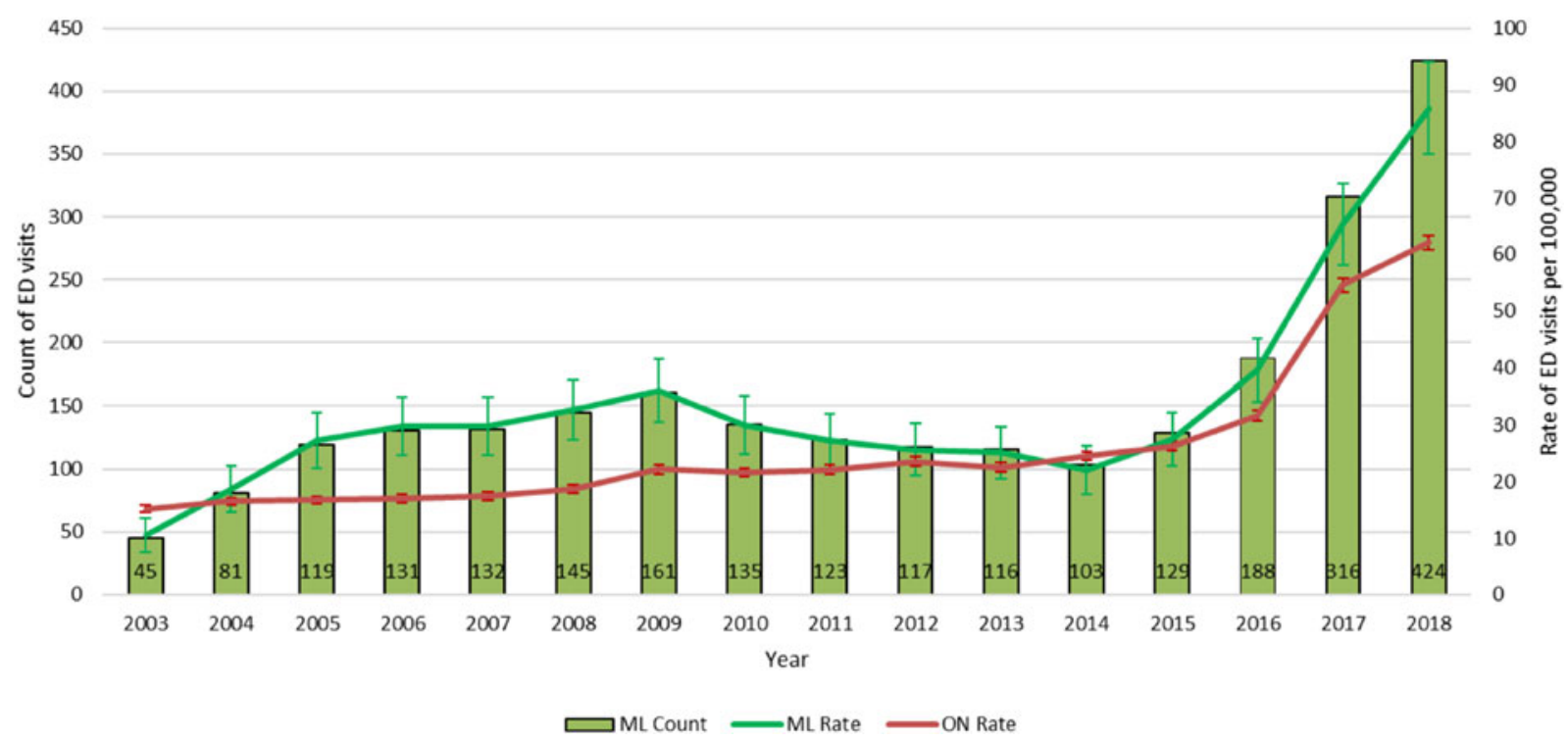

Fig. 3. Rates of emergency department visits related to opioid toxicity in Middlesex-London and Ontario. Data Source: National Ambulatory Care Reporting System (NACRS), 2003-2017, Ontario Ministry of Health and Long-Term Care, IntelliHealth Ontario. Available from https://www.publichealthontario.ca/en/data-and-analysis/substance-use/interactive-opioid-tool\#/trends.

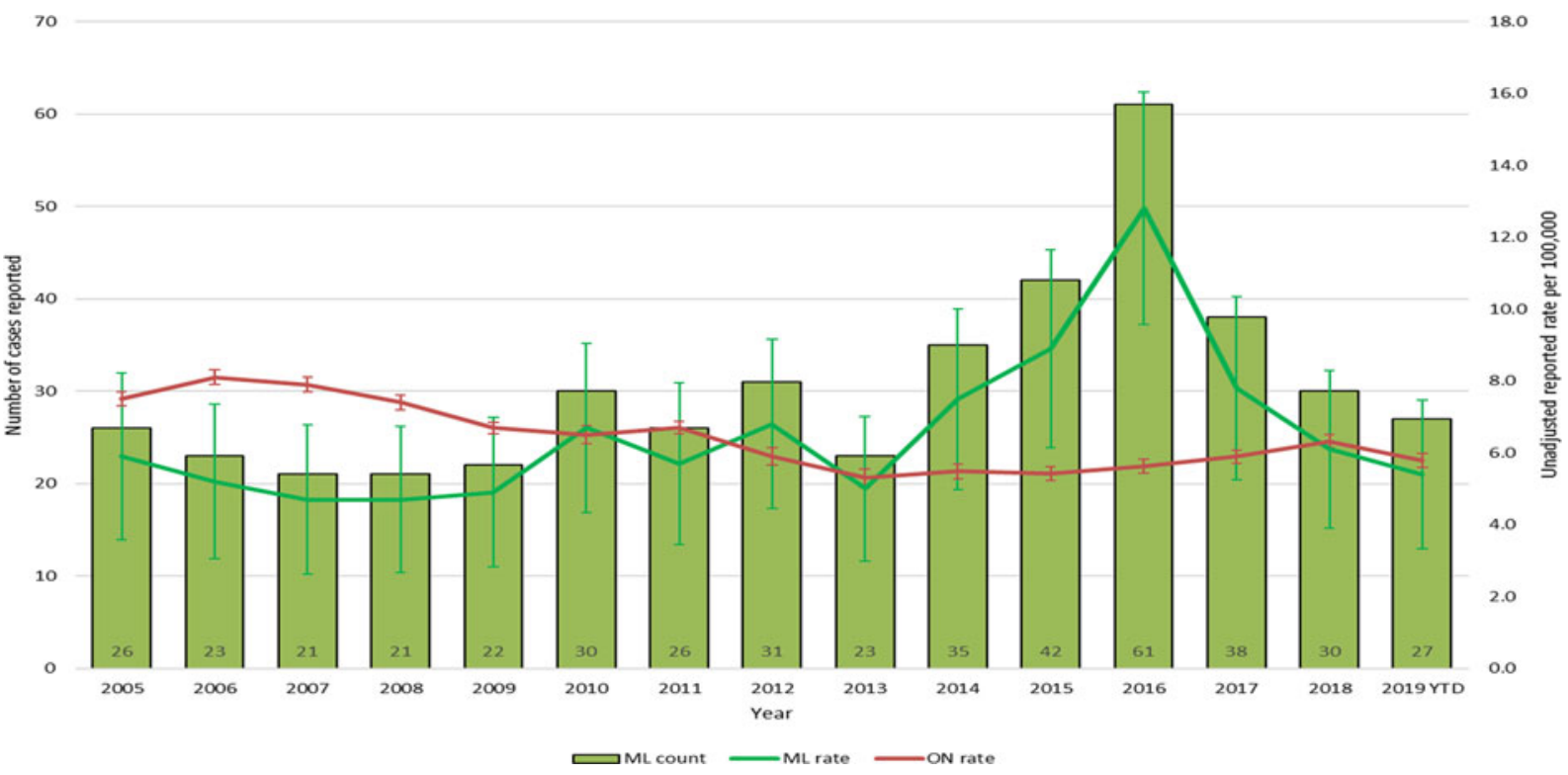

Fig. 4. Count and rate of new HIV cases, Middlesex-London and Ontario, 2005-2019 (Note: Ontario rate excludes Middlesex-London cases). Data source: Public Health Ontario Infectious Disease Query: MLHU: Case counts and crude rates by public health unit and year. Toronto ON: Ontario Agency for Health Protection and Promotion; 2019 Dec 18 [cited 2019 Dec 20]. Available from https://www.publichealthontario.ca/data-and-analysis/infectious-disease/id-query.

During the investigation into the HIV outbreak, it was noted that the case profile was markedly different than that in the rest of the province, or had been locally, prior to the outbreak. In Ontario, HIV rates were declining, and most new cases were identified amongst the population of men who have sex with men. However, the most frequently reported risk factor for new cases associated with the outbreak in London was intravenous drug use [3]. As well, many of the new cases in London were being identified amongst patients seeking care in the hospital ED rather than being identified by family doctors or testing in sexual health clinics [3]. This finding indicated that diagnosis was occurring at a much later stage in the disease than amongst previous cases and that it was likely that these patients were not connected with primary care providers, who may identify symptoms and risk factors earlier. Further investigation confirmed that many of these patients were indeed disconnected from health care, primary or otherwise, and were often not following up with appointments for the treatment of their HIV after they were diagnosed.

Locally, an HIV leadership group was formed comprising the leadership of agencies involved in the care and management of patients with HIV including public health, hospitals, HIV advocacy agencies, addictions support and a community health centre. 


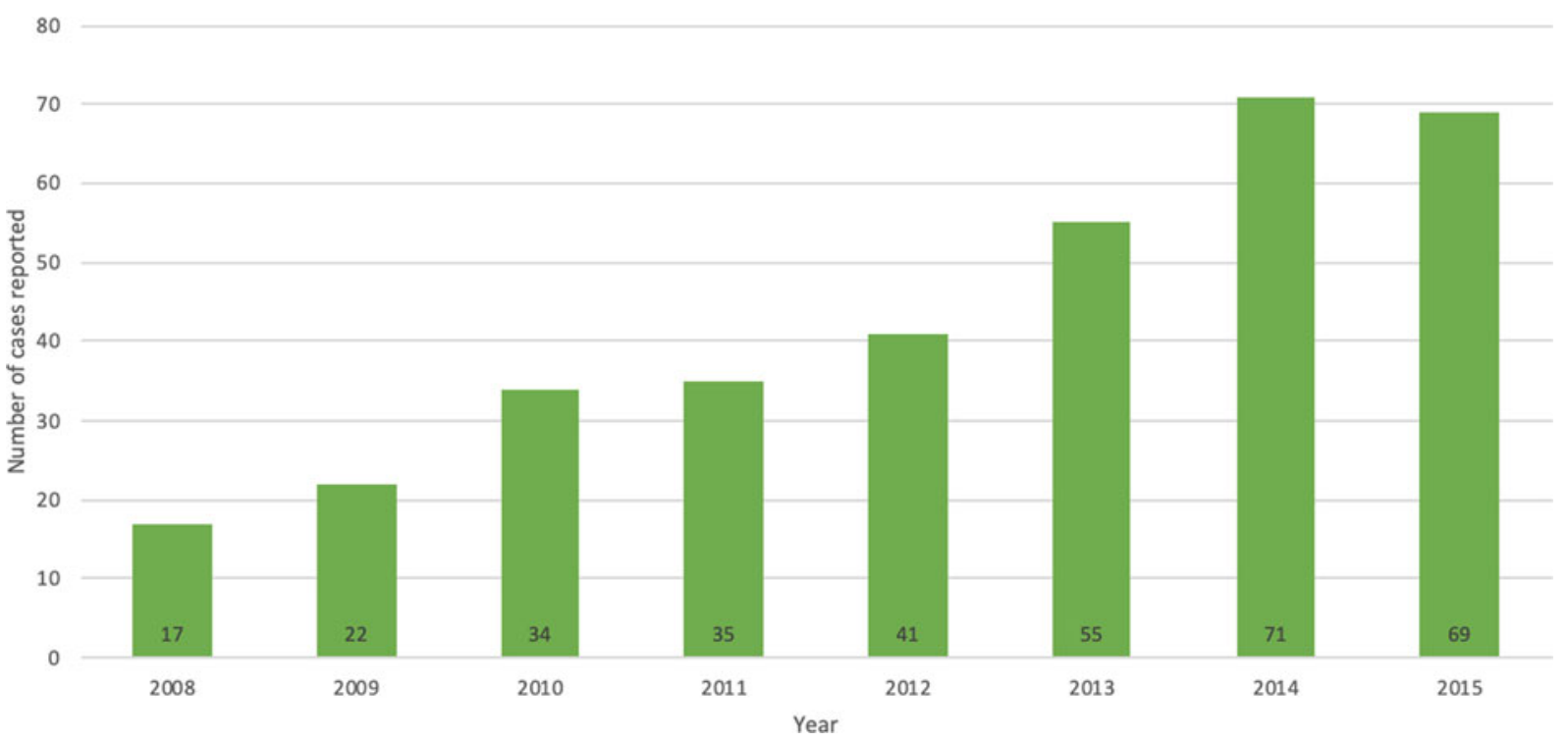

Fig. 5. Count of injection drug use associated endocarditis cases per year admitted to the London Health Sciences Centre. Reproduced courtesy of Dr Michael Silverman MD, FRCP, FACP, Chair/Chief Infectious Diseases, LHSC and St. Joseph's Hospital, Western University, London.

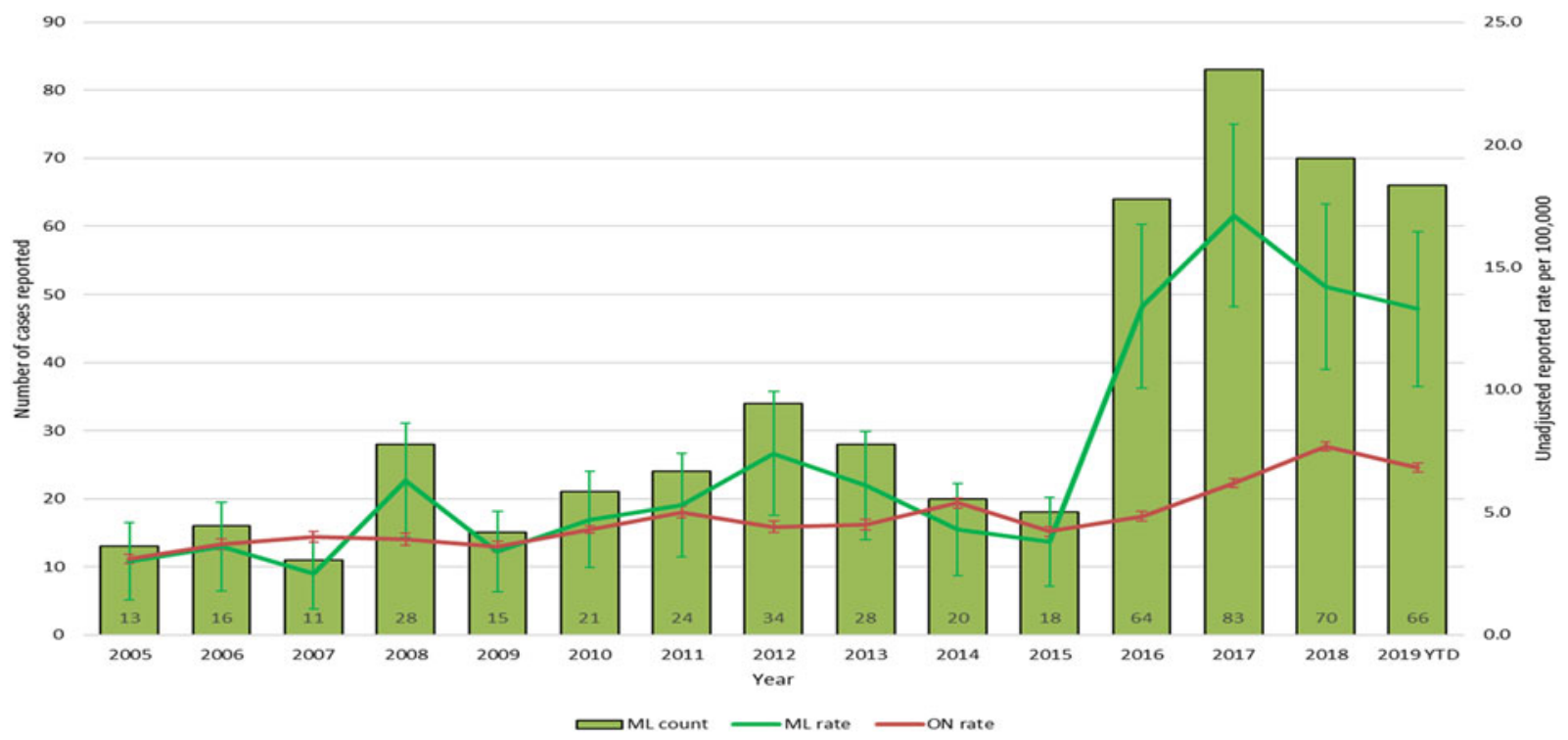

Fig. 6. Count and rate of invasive group A streptococcal (iGAS) infections, Middlesex-London and Ontario, 2015-2019 (Note: Ontario rate excludes Middlesex-London cases). Data source: Public Health Ontario Infectious Disease Query: MLHU: Case counts and crude rates by public health unit and year. Toronto ON: Ontario Agency for Health Protection and Promotion; 2019 Dec 18 [cited 2019 Dec 20]. Available from https://www.publichealthontario.ca/dataand-analysis/infectious-disease/id-query.

The group mapped care pathways for underhoused or homeless patients diagnosed with HIV, identified system gaps and resource constraints, and improved collaboration between agencies for seamless case management. Their work was also helped to establish the province's first sanctioned Temporary Overdose Prevention Site.

Harm reduction measures employed in response to similar HIV outbreaks in Indiana, USA as well as Vancouver and Saskatoon in Canada were studied for potential strategies to be used in Middlesex-London. Numerous traditional harm reduction activities for PWID such as clean needle and equipment distribution programmes had been used in London for several years in response to the growing rates of $\mathrm{HCV}$ and injection drug use in the community. In London, this programme distributes over 3 million needles annually. As a result of their review of harm reduction strategies, MLHU and the Regional HIV/AIDS Connection (RHAC), a local HIV/AIDS support and advocacy agency, further augmented the needle distribution programme to remove some identified barriers by increasing the number of distribution points throughout the city, including local pharmacies and a mobile van. It is believed that these established harm reduction programmes may have prevented earlier arrival of the subsequent outbreaks and may have mitigated their extent.

The characteristics of Vancouver's outbreak were very similar to London's as the primary mode of transmission appeared to be associated with injection drug use [10]. Vancouver Coastal 


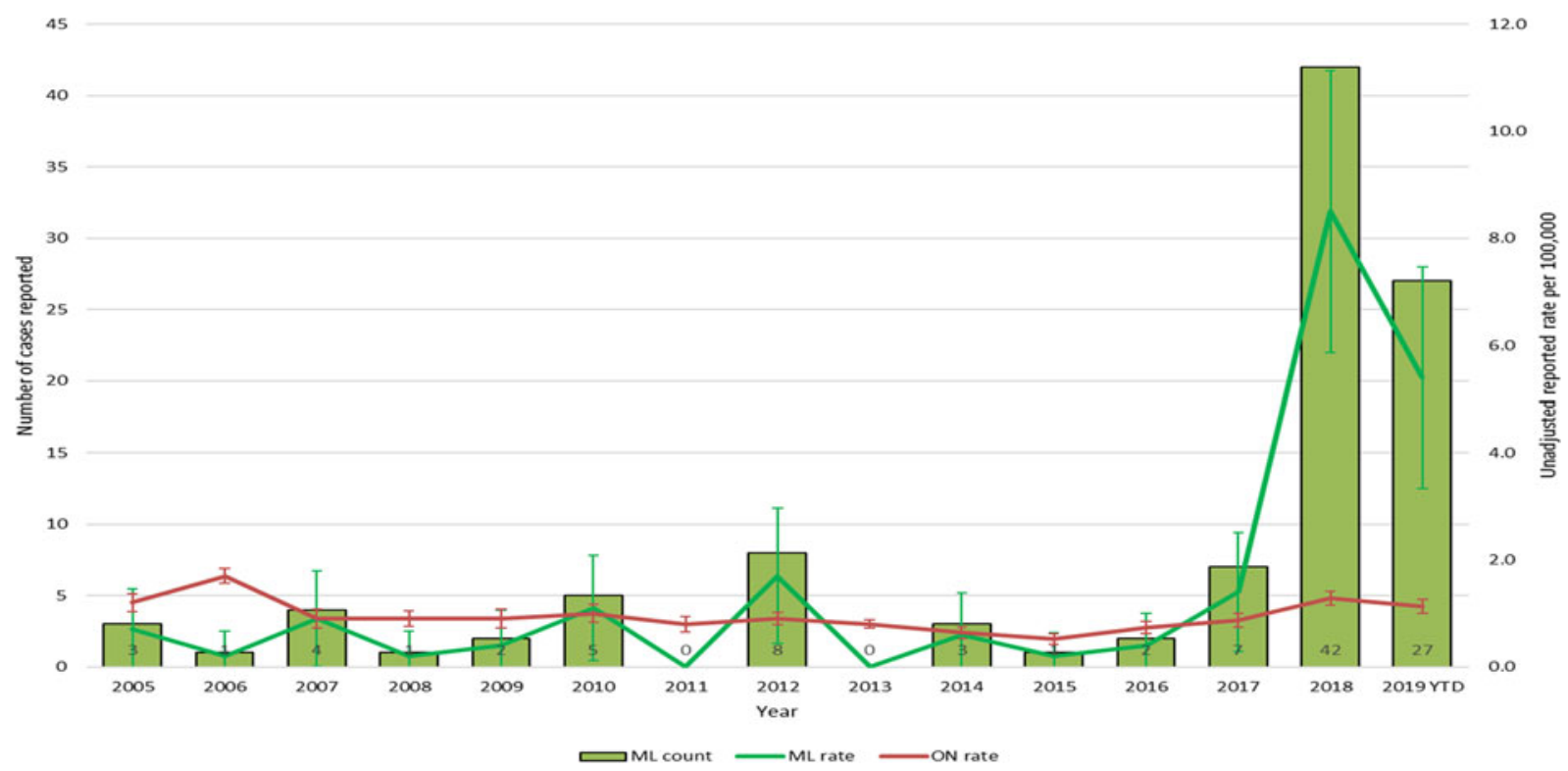

Fig. 7. Count and rate of hepatitis A cases, Middlesex-London and Ontario 2005-2019 (Note: Ontario rate excludes Middlesex-London cases). Data source: Public Health Ontario Infectious Disease Query: MLHU: Case counts and crude rates by public health unit and year. Toronto ON: Ontario Agency for Health Protection and Promotion; 2019 Dec 18 [cited 2019 Dec 20]. Available from https://www.publichealthontario.ca/data-and-analysis/infectious-disease/id-query.

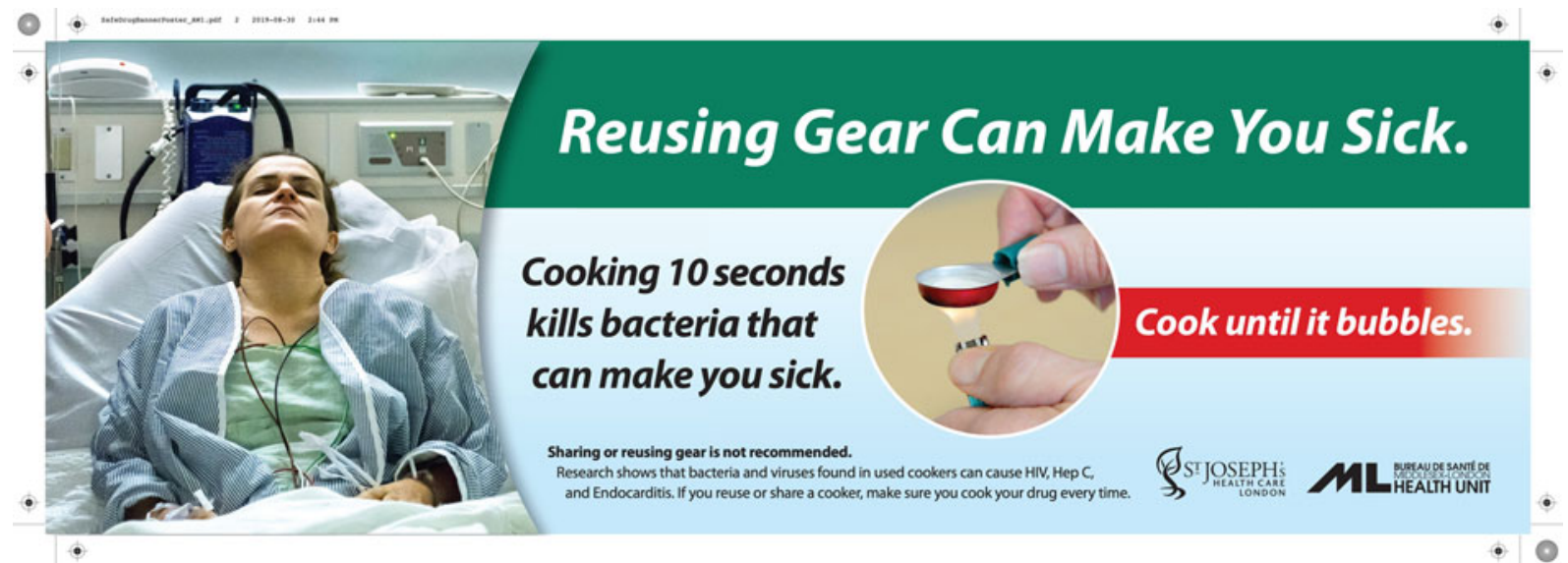

Fig. 8. 'Cook Your Drugs' harm reduction promotional material. Middlesex-London Health Unit. 2020.

Health had developed a comprehensive programme called STOP HIV/AIDS (Seek and Treat for the Optimal Prevention of HIV \& AIDS). Premised on the concept of 'treatment as prevention', the programme involved the deployment of 'pods' of outreach workers and nurses assertively engaging underhoused patients who had recently received an HIV diagnosis. These outreach workers developed relationships with clients over time until the clients were ready to engage in the active treatment of their disease. Vancouver's use of wrap-around care supports provided directly in the community, as opposed to in a hospital environment, was recognized as being instrumental in helping to reduce new cases of HIV in the district by almost 50\% between 2007 and 2016 [11].

The member agencies of the HIV leadership group and staff at MLHU worked to implement key elements of Vancouver's response. MLHU reallocated resources to develop an HIV outreach team comprised of two outreach workers and a public health outreach nurse tasked with establishing connections with people recently diagnosed with HIV but lost to follow-up. Collaborations with local HIV treatment programmes allowed for the development of both hospital- and community-based care pathways and supported referrals from MLHU's outreach team.

All clients of the HIV outreach team were either residing in homeless shelters or 'living rough' on the streets. The outreach team was able to develop care relationships with most clients and engage them directly into ongoing HIV treatment programmes. With intensive and coordinated community efforts, the annual number of new HIV cases reduced by more than $50 \%$ from an all-time high of 63 cases in 2016 to 28 new cases in 2018. Additionally, injection drug use activities had decreased in prevalence amongst new cases of the disease with only $44 \%$ of cases now reporting this risk factor.

In studying the HIV outbreak, local researchers also sought to examine if certain injection drug use techniques might lead to increased risk of transmission of the HIV and/or bacteria 
associated with infective endocarditis. Evidence from that research showed the potential for PWID to reduce their risk of both HIV and endocarditis by heating their drugs to a boil prior to consumption [12-14]. As a result, an awareness campaign targeted to advise PWID to 'Cook Your Drugs' was tested and incorporated into the distribution of harm reduction supplies [Fig. 8]. This campaign was subsequently adopted for provincewide use through the Ontario Harm Reduction Distribution Program.

In October 2018, MLHU alerted health care providers, social agency workers and the general public upon observance of the rapidly increasing rate of new, non-travel-related cases of HAV. Direct email notification was provided to family physicians and social agencies. Posters and leaflets were distributed through homeless shelters, and media releases provided targeted messaging to inform the public and those at highest risk about risk factors and personal protective measures for HAV. Enhanced surveillance was established to further identify risk factors and thorough case and contact follow-up was used to isolate and treat potential cases.

Staff at MLHU immunized a total of 978 close contacts of cases and other at-risk individuals through clinics held at shelters, community meal programmes and the Temporary Overdose Prevention Site. The HIV outreach team served an important role in these efforts as their pre-existing relationships with marginalized clients facilitated higher vaccine uptake within the community. Additionally, inducements of \$5 CAD in gift cards for local coffee shops helped to further increase uptake of the vaccine [9].

The HAV outbreak was declared over in July 2019 after 100 days passed without any additional cases. Robust post-exposure immunization efforts, enhanced communications and strong community partnerships were critical in achieving control of the outbreak.

The local response to the iGAS outbreak leveraged similar strategies as those used for HIV and HAV. Those strategies have included establishing enhanced surveillance to identify those at risk, disseminating targeted communications to healthcare providers and community workers supporting the underhoused, homeless and PWID populations, creating wound care awareness messaging to be distributed with harm reduction supplies, and leveraging the HIV outreach team relationships to ensure those with recent diagnoses of iGAS were connected to appropriate care pathways. Notwithstanding these efforts, the prevalence of iGAS in the community continues to be of concern as rates of the disease remain high in both marginalized and nonmarginalized populations.

Although Canada provides universal health care to its residents, many of those who are homeless or have addictions are still reluctant to seek treatment for medical concerns. This may be due to stigmatization or a loss of trust in care providers and authorities, but the result appears to be an exacerbation of the impacts of treatable and preventable health threats such as infectious disease. While the outbreaks discussed were not limited to marginalized populations, the burden of illness has disproportionately impacted those who were underhoused or used injection drugs.

Enhanced surveillance methods including genotyping and extended case and contact tracing were used in these investigations but, since the outbreaks were investigated independently, data limitations were noted that impaired the ability to draw clear linkages between the various outbreaks. Considerations to address this gap could include more detailed questionnaires to better identify and delineate common risk factors, crossreferencing to identify cases involved in more than one outbreak investigation, and examining the role migration plays in the introduction of these diseases into the community.

The establishment of robust working relationships between public health and community service agencies who support marginalized populations has been viewed as a crucial component in responding to these outbreaks. These relationships served to help strengthen care pathways for clients who are prone to be disconnected from care, especially through the development of compassionate connections between clients and health/social agency workers.

As previously noted, the outreach team developed to respond to the HIV outbreak in London was tasked to also play a key role in the response to the HAV and iGAS outbreaks as they had built trust relationships with people experiencing homelessness. As a result, MLHU has considered broadening the HIV outreach team's mandate to support infectious disease control amongst people experiencing homelessness. The success of these programmes in aiding the response to the outbreaks highlights the need for public health and primary care to establish ongoing street-level engagement so they can provide rapid and effective outbreak interventions, prevent future outbreaks and help ensure better health outcomes for vulnerable populations. Further, the efforts involved in addressing each of these outbreaks were substantial.

Bobby Milstein at the Centres for Disease Control and Prevention identified that it is essential to address the underlying common elements that allow these outbreaks to take root in order to resolve a syndemic. 'To prevent a syndemic, one must not only prevent or control each disease but also the forces that tie those diseases together.' [15]

The infection control challenges posed by shared injection drug equipment, poor sanitary conditions and the crowded congregate living areas of homeless shelters will continue to create a large risk of similar outbreaks occurring in the future. Stronger collaboration between health authorities and social service agencies to provide street-level health supports, robust harm reduction programmes and housing prioritization are seen as crucial to lessen the risk of future outbreaks and interrupt chains of disease transmission.

Acknowledgements. Alexander Summers, Alison Locker, Sarah Maaten, Michael Silverman, Linda Turner provided advice and data for this manuscript.

Financial support. This activity received no specific grant from any funding agency, commercial or not-for-profit sectors.

Conflict of interest. None.

\section{References}

1. Statistics Canada. Census profile, 2016 census, London, City. Available at https://www12.statcan.gc.ca/census-recensement/2016/dp-pd/prof/details/page. cfm? Lang $=$ E\&Geo1 $=$ CSD $\&$ Code1 $=3539036 \& G e o 2=$ PR\&Code2 $=35 \&$ SearchText $=$ London\&SearchType $=$ Begins $\&$ SearchPR $=01 \& \mathrm{~B} 1=$ All $\&$ GeoLevel $=$ PR\&GeoCode $=$ 3539036\&TABID=1\&type $=0$. Accessed 6 September 2019.

2. City of London. Counting our way home 2018 enumeration event results. Available at https://www.london.ca/residents/homeless-prevention/ Documents/171030028-COL-Enumeration-Event-2018-Summary-ReportEMAIL-WEB.pdf. Accessed 6 September 2019.

3. Middlesex-London Health Unit. Development and implementation of a strategy to address HIV, hepatitis C, invasive group A streptococcal disease and infective endocarditis in persons who inject drugs in 
Middlesex-London, Ontario. Available at https://www.healthunit.com/ uploads/2017-04-20-report-021-17.pdf. Accessed 10 March 2020.

4. Public Health Agency of Canada. Canadian Tuberculosis Standards 7th Edition: 2014 - Chapter 10: Tuberculosis and Human Immunodeficiency Virus. Available at https://www.canada.ca/en/publichealth/services/infectious-diseases/canadian-tuberculosis-standards-7th-edition/ edition-6.html. Accessed 13 March 2020.

5. Singer M and Clair S (2003) Syndemics and public health: reconceptualizing disease in bio-social context. Medical Anthropology Quarterly 17, 423-441.

6. American Public Health Association (2000) Viral hepatitis C. In Chin J and Ascher M (eds), Control of Communicable Diseases Manual, 17th Edn. Washington: American Public Health Association, pp. 252

7. Middlesex-London Health Unit. MLHU to address local public health emergency. Available at https://www.healthunit.com/news/mlhu-toaddress-local-public-health-emergency. Accessed 5 December 2019.

8. Middlesex-London Health Unit. Summary information report, April 2017. Available at https://www.healthunit.com/uploads/2017-04-20report-022-17.pdf. Accessed 10 March 2020.

9. Middlesex-London Health Unit. Hepatitis A outbreak declared over. Available at https://www.healthunit.com/uploads/2019-10-17-report-06419.pdf. Accessed 10 November 2019.
10. Gilbert M, Buxton J and Tupper $\mathbf{K}$ Decreasing HIV infections among people who use drugs by injection in British Columbia: Potential explanations and recommendations for further action - 2011. Report from the Office of the Provincial Health Officer. Vancouver: Office of the Provincial Health Officer.

11. BC Centre for Disease Control HIV in British Columbia: Annual Surveillance Report - 2016. Vancouver, BC, Canada.

12. Weir M, et al. (2019) The risk of infective endocarditis among people who inject drugs: a retrospective, population-based time series analysis. Canadian Medical Association Journal 191(4), E93-E99. Published online: 28 January 2019. doi: doi:10.1503/cmaj.180694.

13. Ball L, et al. (2019) Sharing of injection drug preparation equipment is associated with HIV infection: a cross sectional study. Journal of Acquired Immune Deficiency Syndrome 81(4), e99-e103. Published online: 1 August 2019. doi: 10.1097/QAI.0000000000002062.

14. Ball L, et al. (2019) Heating injection drug preparation equipment used for opioid injection may reduce HIV transmission associated with sharing equipment. Journal of Acquired Immune Deficiency Syndrome 81(4), e127-e134. doi: doi:10.1097/QAI.0000000000002063.

15. Milstein B (2002) Introduction to the Syndemics Prevention Network. Atlanta, GA: Centers for Disease Control and Prevention. 